\title{
ESTRATEGIAS FAMILIARES PARA EL TRÁNSITO A LA VIDA ACTIVA DE LA JUVENTUD RURAL: MODELOS DE INSERCIÓN SOCIOLABORAL ${ }^{1}$
}

\section{Cecilia Díaz Méndez}

Universidad de Oviedo

E-mail: cdmendez@hp845.econo.uniovi.es

\begin{abstract}
RESUMEN
El presente trabajo se apoya en dos soportes teóricos fundamentales: por un lado, la corriente teórica que en Sociología Rural se conoce como Estudios Campesinos; por otro lado, el estudio de las estrategias familiares en las familias campesinas. La investigación muestra cómo los padres elaboran estrategias de acción para orientar social y laboralmente el futuro de sus hijos e hijas, y esta orientación está determinada por el objetivo de continuidad de la explotación familiar de la que depende el grupo. A partir de este análisis se elaboran modelos de inserción sociolaboral de los jóvenes y las jóvenes y aparecen los factores que determinan que cada hijo/a se oriente en una u otra dirección. Este trabajo se ha desarrollado en la región asturiana y está basado en una metodología de tipo cualitativo con entrevistas en profundidad y grupos de discusión.
\end{abstract}

\section{INTRODUCCIÓN: ASPECTOS TEÓRICOS EN EL ANÁLISIS DEL TRÁNSITO DE LA JUVENTUD RURAL A LA VIDA ACTIVA}

El objetivo del presente trabajo es explicar los componentes y determinantes de los procesos de transición de la juventud rural a la vida adulta y realizar una descripción de estas trayectorias de inserción. Para ello comenzamos

1 Este trabajo forma parte de la tesis doctoral de la autora, que ha sido financiada a través de las becas de Formación de Personal Investigador del Ministerio de Educación y Ciencia y publicada por el Ministerio de Agricultura, Pesca y Alimentación (Díaz Méndez, 1997). 
con un repaso de las particularidades de este proceso entre la juventud vinculada por su origen a la agricultura familiar. Continuamos con un análisis del comportamiento estratégico familiar, comportamiento decisivo para comprender las formas de inserción social y laboral de los jóvenes y las jóvenes de estas familias. A continuación se explica la metodología empleada en la investigación y se finaliza con los modelos de tránsito a la condición de adulto que sigue la juventud rural.

\subsection{LA TRANSICIÓN A LA VIDA ADULTA DE LA JUVENTUD RURAL}

Las investigaciones que explican el tránsito de los jóvenes a la vida adulta muestran las dificultades de aplicar el concepto de transición a una juventud que procede de familias campesinas. Este proceso de transición ha sido estudiado en nuestro país con jóvenes urbanos. En estos trabajos se considera que la inserción se inicia con la entrada en el primer empleo que, tras diferentes etapas, llevará a la juventud al logro de una autonomía económica, de una autonomía en el espacio (vivienda propia) y a la formación de una familia propia (Zárraga, 1985; Zárraga y Conde, 1985; Casal, Masjuan y Planas, 1991). Los escasos trabajos sobre la juventud rural existentes en España indican que ésta no es la pauta seguida por la juventud rural y que la inserción social y laboral cuenta con particularidades, al menos, en tres aspectos: la formación, el empleo y la independencia espacial de la familia de origen ${ }^{2}$.

En primer lugar hay que reseñar los aspectos formativos; concretamente un menor acceso a la enseñanza reglada en comparación con la juventud urbana y un mayor fracaso escolar y abandono de los estudios, hacen que la formación no sea un elemento diferenciador entre los rurales ni determinante de su inserción laboral, como ocurre en el mundo urbano ${ }^{3}$.

En segundo lugar es preciso reconocer las particularidades del trabajo agrario familiar, un trabajo en el que se encuentran vinculados todos los miembros de la familia, aprendido prioritariamente a través del proceso de socialización primaria y realizado en un entorno laboral en el que los roles determinan la vinculación de los miembros jóvenes al trabajo y su implicación laboral concreta. La mayor parte de los jóvenes agrarios españoles se encuentran en la

2 No existe un estudio específico sobre la juventud rural hasta 1984 (González, De Lucas y Ortí, 1985), aunque ya la primera Encuesta de Juventud en 1960 tuvo en cuenta los distintos tipos de hábitats de residencia de la juventud (Gabinete de Estudios Juveniles, 1960) y la Encuesta de Juventud de 1982 (Toharia y García Ferrando, 1982) incluyó en su estratificación a los residentes en núcleos de población con menos de 2.000 habitantes.

${ }^{3}$ La probabilidad de logro educativo de los hijos e hijas de los agricultores se ha multiplicado en los últimos años (han mejorado particularmente las cohortes más jóvenes); no obstante, siguen ocupando las peores posiciones educativas, junto con los hijos de jornaleros (González y Gómez Benito, 1997). 
categoría laboral de ayudas familiares, y muchos de ellos lo serán durante toda su vida laboral, en particular las mujeres ${ }^{4}$.

En tercer lugar debemos considerar las particularidades de la independencia de la familia de origen, que hace que nos encontremos con jóvenes que nunca se independizan del grupo familiar (los sucesores en la explotación agraria) y jóvenes que lo hacen y se desvinculan totalmente del grupo (Díaz Méndez, 1997).

Estos antecedentes en la formación, en el trabajo y en la independencia espacial hacen de la juventud rural un grupo que debe ser analizado a partir de la consideración de estas particularidades; y se trata de características que proceden de su vínculo con una familia ligada al trabajo en el campo.

\subsection{LA REPRODUCCIÓN EN LAS FAMILIAS CAMPESINAS}

Las familias campesinas han sobrevivido a lo largo del tiempo hasta la actualidad como una forma específica de producción en la que familia y explotación constituyen una unidad inseparable. Si bien los cambios sociales les han afectado de forma significativa, modificando aspectos decisivos de su forma de vida, estos cambios no han sido suficientes para acabar con un grupo cuyo objetivo es su propia reproducción y caracterizado por su capacidad de adaptación (Newby y Sevilla Guzmán, 1983; Shanin, 1976).

En las familias campesinas tradicionales, un rígido comportamiento del grupo permitía al individuo un escaso margen de maniobra en la toma de decisiones. Para lograr su reproducción, el grupo controlaba rígidamente el comportamiento de sus miembros haciendo coincidir sus intereses individuales con los familiares (Chayanov, 1974; Galeski, 1977).

La familia campesina actual sigue siendo la responsable de la supervivencia del grupo; de hecho, la explotación sobrevive si la familia sobrevive. No ha variado su objetivo y busca reproducir o mejorar sus condiciones de vida y trabajo. Pero las familias campesinas modernas pasan por dificultades para lograr su reproducción ya que el entorno, en constante cambio, no les es favorable. Muchas familias/explotaciones desaparecen ya que los elementos que garantizaban el éxito de sus estrategias reproductivas en el pasado han variado sustancialmente (Palerm, 1980; Sevilla Guzmán, 1984).

Considerando aquellos elementos que definen el comportamiento estratégico (Garrido Medina y Gil Calvo, 1993), se pueden reseñar algunas de estas dificultades en el caso de las familias campesinas:

a) El objetivo estratégico de la familia campesina es, y sigue siendo, reproducirse como grupo. La reproducción en este tipo de familias pasa por las

4 Para un repaso actualizado y desde diversas perspectivas sobre la situación de la agricultura familiar en España se puede ver Bretón, García Pascual y Mateu González (1997). 
condiciones de la producción. El éxito se consigue si el grupo cuenta con un sucesor que continúe el trabajo agrario familiar. Pero tanto la explotación como las posibilidades de lograr un sucesor están amenazadas en las familias modernas. Por un lado, la modernización pone en cuestión la eficacia de las explotaciones familiares y con ello la sucesión. Si en el pasado era un bien deseable como futuro para los jóvenes, hoy no resulta atractivo para la juventud (González, 1993).

b) Los recursos han variado, en particular los financieros, técnicos y económicos de la explotación, ofreciéndose a la juventud con un nuevo valor (Lisón, 1971; González y Gómez Benito, 1997). Además, junto a los recursos propios, limitados en las familias tradicionales y también en las modernas, existen recursos externos nuevos susceptibles de ser utilizados por las familias, básicamente las posibilidades de formación y empleo no agrario que ofrece el entorno. Estos recursos tácticos hacen que el margen de maniobra para actuar sea mayor en las familias modernas y las familias lo usan a su favor (Carabaña, 1993).

c) La incertidumbre en el entorno general ha aumentado; las respuestas rutinarias del grupo familiar para actuar, que antes tuvieron éxito, resultan ahora inapropiadas. Los hábitos adquiridos por tradición, tanto productivos como familiares, se modifican al errar en la acción.

d) Los actores que intervienen en la toma de decisiones también han variado. Los actores internos, los propios miembros del grupo, interactúan en un entorno familiar en el que la rigidez normativa, marcada por la estructura jerárquica de la familia tradicional, se cuestiona o se encuentra difuminada. Si en las familias tradicionales se supeditaban los intereses grupales a los individuales, esto no resulta tan evidente en las familias modernas, donde los intereses individuales no siempre coinciden con los del grupo y en los que la juventud cobra protagonismo. Por otra parte, los actores externos al grupo interfieren de una forma más determinante que en el pasado en las decisiones familiares, como se observa en los cambios provocados por las acciones políticas que afectan al sector agrario europeo (Moyano Estrada y Fernández Durántez, 1990; González, 1990).

La familia, en este contexto, organiza sus recursos y los hace variar para adaptarse a las exigencias y necesidades de la sociedad en la que está inmersa, adopta estrategias de acción en un nuevo entorno para perseguir un objetivo tradicional, su pervivencia como grupo. En unos casos, las familias logran su objetivo reproductor y uno de sus hijos prosigue con el trabajo familiar; en otros casos fracasan y no encuentran un heredero que reproduzca su historia familiar. Existen también situaciones en las que, con un aparente éxito, las estrategias de acción les llevan a una muerte irreversible aun a pesar de contar con un hijo continuador.

Las estrategias de acción seguidas por la familia no son respuestas en el vacío; se trata de un comportamiento que se elabora en un entorno que cuenta 
con factores que orientan la acción estratégica familiar. Entre otros factores, cabría resaltar al menos tres de ellos.

Habría que considerar en primer lugar las leyes hereditarias, puesto que obligan a adoptar una determinada conducta estratégica para lograr la reproducción. Aunque la orientación de estas leyes consuetudinarias haya cambiado, el carácter hereditario del patrimonio agrícola orienta una determinada conducta de reproducción y un manejo específico y predeterminado de los recursos (Contreras, 1997).

En segundo lugar, las investigaciones sobre la organización familiar apuntan a la calidad y a la cantidad de los recursos humanos (hijos e hijas), básicamente la edad y el género y las relaciones parentales dentro del grupo, como factores determinantes para entender las estrategias familiares (Carabaña, 1993). De forma particular se considera que la familia campesina organiza su actividad productiva en función de sus recursos humanos, hasta tal punto que la organización social del grupo sigue un fuerte paralelismo con su organización económica (Segalen, 1980; Palerm, 1980).

En tercer lugar, debe considerarse que la familia toma sus decisiones en un momento concreto de su ciclo vital. Así, las estrategias variarán en función de la composición del grupo y la edad de los miembros, adaptándose los cambios en la explotación a los cambios relevantes del grupo familiar (matrimonio, jubilación, etc.).

Las familias, ante estas variadas y cambiantes circunstancias, deciden qué hacer, qué no hacer, cuándo, cómo, con quién...; evalúan la conveniencia de adoptar unas $\mathrm{u}$ otras decisiones. Lo que destaca en este comportamiento estratégico es la adaptabilidad de las familias campesinas para responder a un entorno no siempre favorable o claramente adverso, usando sus recursos o utilizando a su favor los del entorno para desarrollar sus estrategias reproductivas.

\section{METODOLOGÍA}

Las estrategias familiares reproductivas han sido analizadas frecuentemente a través de sus resultados, el cierre o la continuidad de las explotaciones agrarias, los cambios en las explotaciones o en las familias que las sustentan; pero se sabe poco acerca de cómo la familia maneja sus recursos y posibilidades de acción para tomar una decisión. Probablemente se ha obviado este proceso, no por carecer de importancia, sino por tratarse de un fenómeno que sucede en el entorno íntimo del grupo y que es el resultado de la interacción continuada de todos sus miembros. Este proceso cuenta con particularidades que le hacen difícilmente abordable y que orientan la investigación hacia un análisis de carácter cualitativo.

En primer lugar, se trata de un proceso en el que el objetivo estratégico no siempre es consciente; incluso a veces es cambiante, contradictorio o incierto para el grupo familiar. Se trata de una orientación que se produce a lo largo de 
la vida de los individuos y en la que, de un modo activo, van dándose variaciones a medida que el grupo y la explotación van cambiando.

En segundo lugar, estamos hablando de un comportamiento de tipo colectivo, de tal modo que, si bien es un proceso que afecta al individuo en particular (es el joven o la joven quien se ve afectado), depende del grupo familiar en su conjunto y el propio grupo se ve afectado por él. Si estudiamos el comportamiento individual, las decisiones colectivas, en particular los conflictos producto de la interacción, pueden pasar desapercibidas e impiden conocer el problema en profundidad. No olvidemos, además, que se trata de unas familias en las que la interdependencia, tanto social como laboral, las particulariza como grupo.

En tercer lugar, se trata de un proceso que por ser colectivo y orientador puede verse, a ojos de sus participantes más jóvenes, como socialmente indeseable. El hecho de que las familias adopten estrategias para lograr una particular inserción de sus hijos confirma una cierta manipulación del grupo familiar sobre el joven. Esto podría llevar a difuminar la intervención parental en el proceso y volverse intencionadamente oculto.

En cuarto lugar, hay que recordar que se trata de un grupo que se encuentra en un momento de cambio y adaptación, los sucesos intrafamiliares y extrafamiliares pueden hacer variar la orientación de los padres hacia sus hijos, los cambios no se estructuran con facilidad por estar produciéndose en el momento en que se están estudiando.

Todas estas particularidades dificultan el análisis y nos conducen a una forma diferente de afrontar el problema; para conocer los procesos seguidos por el grupo familiar para decidir sobre el futuro de sus hijos e hijas es preciso hacerlo desde una metodología de trabajo abierta, capaz de entender al sujeto tal y como él mismo se percibe en el medio. Para ello se ha optado por una investigación que haga hablar a los protagonistas. Se considera que el lenguaje natural es autorreflexivo, que las personas hablan sobre sí mismas y al hacerlo suplantan la realidad simulándola con el lenguaje: «las palabras se ponen en el lugar de las cosas, los discursos se ponen en el lugar del mundo" (Ibáñez, 1979: 131).

Interesa que sean los propios protagonistas quienes nos lo cuenten; se trata de un proceso interno del grupo familiar y el investigador debe interferir lo menos posible para que ellos hablen libremente, que manifiesten sus deseos y creencias, que emitan un discurso subjetivado. No se espera un discurso objetivo, ni tampoco interesa, para evitar la estructuración de una realidad que es formalizada por los propios actores.

Trabajemos con entrevistas en profundidad y con grupos de discusión ${ }^{5}$.

5 Estas técnicas se iniciaron en España en 1965 a partir de los trabajos de Ibáñez y sus discípulos. Las prácticas metodológicas desarrolladas por ellos dieron lugar a lo que se llamó la Escuela madrileña de cualitativistas (Ortí, 1986). Este grupo ha realizado numerosas investigaciones de tipo académico, aunque esta metodología tuvo y tiene su mayor aplicación en la investigación de mercados. 
Esta elección parte de considerar estos instrumentos decisivos para hacer que el individuo se convierta en una fuente de información sobre sí mismo y sobre las personas que le rodean. Las entrevistas permiten averiguar las razones que mueven su comportamiento y la percepción e interpretación que los protagonistas tienen de él. Los grupos ponen en contacto a sujetos que por sus particularidades son un reflejo del grupo social que se quiere analizar, reproduciendo el conjunto y la diversidad de discursos sociales existentes sobre el objetivo de la investigación.

Para el trabajo se ha seleccionado una tipología de familias agrarias en un área geográfica del norte de España (Asturias). La elección de las familias se produjo, en primer lugar, a partir de criterios basados en los recursos agroganaderos de las explotaciones, siguiendo los registros realizados por la Administración regional en los que se da cuenta de los aspectos productivos más relevantes de las explotaciones familiares agrarias de la región (SADEI, 1994). Esta información de carácter descriptivo se completó con los análisis sobre el dinamismo económico de las explotaciones a partir de elementos de carácter económico y geográfico (Pérez Rivero, 1991; Rodríguez Gutiérrez, 1991). Esta documentación permitió establecer una tipología de explotaciones agrarias que representa la diversidad agraria regional: explotaciones modernizadas con dedicación exclusiva, agricultura de subsistencia con dedicación exclusiva, agricultura a tiempo parcial combinada con minería, agricultura a tiempo parcial combinada con industria.

El paso siguiente fue determinar las áreas en las que seleccionar lar familias de estudio. Para ello se consideró la oferta formativa de la zona, como recursos externos. Se utilizó la información sobre recursos educativos del Ministerio de Educación y Ciencia en la región (Ministerio de Educación y Ciencia, 1991). Se complementó con información sobre el mercado local y las condiciones sociolaborales de los jóvenes rurales asturianos a partir de los trabajos sobre empleo juvenil realizados en la región por la Consejería de la Juventud (Consejería de la Juventud, 1989a, 1989b, 1991).

Siguiendo estos criterios se distinguen cuatro tipos de familias:

Un primer tipo de familias vinculadas a una agricultura moderna y con plena dedicación. Explotaciones con alto nivel de mecanización, de orientación láctea. Con alta productividad respecto a la media regional y regentadas por los titulares más jóvenes de la región. Se ubican en zonas en las que existe una alta concentración de ofertas formativas agrarias. Se seleccionaron en ella seis familias y se realizan veintinueve entrevistas y cuatro grupos de discusión (familias tipo A).

Un segundo grupo de familias vinculadas a explotaciones de pequeñas dimensiones y poca carga ganadera, con bajos niveles técnicos y escasa productividad. Están situadas en zonas de dificultades geográficas (pendientes acusadas, relieves abruptos) y donde el retroceso de la actividad agraria en los últimos diez años ha sido mayor que la media regional. Están regidas por jubilados o personas de edad avanzada, siendo frecuente que las pensiones de jubila- 
ción constituyan un complemento de ingresos de una actividad económica de subsistencia. Las rentas familiares son bajas, cuentan con pocos jóvenes en la zona y con escasas o nulas ofertas formativas, ni especializadas ni generales. En ellas se ha entrevistado a ocho familias, un total de cuarenta y una entrevistas y tres grupos de discusión.

Un tercer grupo de familias con explotaciones agrarias vinculadas laboralmente a la minería que tienen posibilidades en el entorno de acceso a ofertas de formación no reglada. Es un tipo de agricultura que ha existido en la región desde los inicios de la industrialización. Las explotaciones se mantienen hoy a nivel de subsistencia y constituyen un soporte ante las cada vez más frecuentes situaciones de crisis del sector minero regional. Se trata de explotaciones de pequeñas dimensiones situadas en enclaves mineros, envejecidas y con escasa presencia de jóvenes. El descenso de estas explotaciones, aunque ha sido importante, es menor que el del grupo anterior. En este área se ha entrevistado a siete familias, un total de vienticinco entrevistas y cuatro grupos de discusión.

Y un cuarto y último área que cuenta con una agricultura moderna que se compatibiliza con el trabajo en la industria y cuyas explotaciones están ubicadas en unas zonas cuya oferta formativa es muy variada y la más amplia de la región. Son explotaciones en las que el titular tiene un empleo externo en la industria. Encuentra dificultades para su expansión agraria por la competencia en el uso del suelo por parte de la industria. Son explotaciones de medianas dimensiones, muy tecnificadas, que han crecido en los últimos años y con un relativo margen de rentabilidad. Aquí se entrevistó a siete familias, un total de treinta y tres entrevistas y tres grupos de discusión.

\section{ESTRATEGIAS FAMILIARES: VARIEDAD Y CONFIGURACIÓN}

Los resultados del trabajo permiten confirmar que la familia utiliza estrategias colectivas de acción para lograr su reproducción, y esta reproducción se sostiene a través de la orientación del tránsito de los miembros jóvenes del grupo a la vida activa. Se pueden distinguir en el estudio tanto la variedad de estrategias como sus determinantes y la forma en que estas estrategias se configuran.

El concepto de estrategia familiar y el concepto de reproducción asociado a la familia campesina encuentran su conexión en los tres elementos que definen el comportamiento estratégico: En primer lugar, el uso de recursos materiales con que cuenta la familia para maniobrar en el logro de sus objetivos, representados aquí por las características de las explotaciones agroganaderas y las condiciones formativas del entorno. En segundo lugar, la orientación familiar hacia unos objetivos comunes que se polarizan entre proseguir o no con la actividad laboral de la familia. En tercer lugar, la orientación se precisa en las relaciones entre los actores, que dan como resultado una variedad de estrategias de transición a la vida activa de los jóvenes de la familia. 
La familia campesina se ve obligada a realizar una doble selección para hacer posible su objetivo de reproducción. En una primera selección el grupo familiar organiza sus recursos buscando un sucesor. Esto hace que las distintas trayectorias de vida de los jóvenes y las jóvenes se diferencien en función de su continuación en la explotación familiar o de su ruptura con ella.

En función de la disponibilidad de recursos la familia realiza una segunda selección. El número, la edad y el sexo de los miembros jóvenes del grupo son los factores que intervienen en ella. Esta selección actúa sobre los hijos e hijas, el elemento humano. Las condiciones económicas de las explotaciones constituyen el elemento económico. El heterogéneo entorno determina las posibilidades de acceso a recursos formativos y laborales no agrarios que son utilizados por la familia para ofrecer a los hijos e hijas que no prosiguen en la familia $y$ favorecer su tránsito a la vida activa fuera de ella.

Podemos concretar las diferentes trayectorias de transición a la vida activa seguidas por los jóvenes y las jóvenes en el siguiente cuadro, que refleja las variables familiares y de orientación que intervienen en ella ${ }^{6}$ :

\section{Tipologías familiares para el tránsito a la vida activa de la juventud rural según tipo de familia y orientación familiar}

\begin{tabular}{|c|c|c|}
\hline \multirow[b]{2}{*}{ Tipos de familia } & \multicolumn{2}{|c|}{ Orientación familiar } \\
\hline & Trayec. de continuación & Trayec. de no continuación \\
\hline $\begin{array}{l}\text { Familia tipo } A \\
\text { Agricultura familiar moderna y } \\
\text { ofertas formativas agrarias }\end{array}$ & $\begin{array}{l}\text { Absorción masculina } \\
\text { Absorción femenina }\end{array}$ & $\begin{array}{l}\text { Expulsión } \\
\text { Estudiante } \\
\text { Matrimonial de desarraigo }\end{array}$ \\
\hline $\begin{array}{l}\text { Familia tipo } B \\
\text { Agricultura de subsistencia y es- } \\
\quad \text { casa oferta formativa }\end{array}$ & $\begin{array}{l}\text { Sucesión afectiva } \\
\text { Femenina de retorno } \\
\text { Trayectoria puente }\end{array}$ & $\begin{array}{l}\text { Expulsión } \\
\text { Estudiante } \\
\text { Matrimonial de desarraigo }\end{array}$ \\
\hline $\begin{array}{l}\text { Familia tipo } C \\
\text { Agricultura de campesinos mi- } \\
\text { neros y con ofertas de forma- } \\
\text { ción no reglada }\end{array}$ & & $\begin{array}{l}\text { Nostalgia } \\
\text { Matrimonial de desarraigo } \\
\text { Estudiante }\end{array}$ \\
\hline $\begin{array}{l}\text { Familia tipo } D \\
\text { Agricultura a tiempo parcial y } \\
\text { concentración de ofertas for- } \\
\text { mativas }\end{array}$ & $\begin{array}{l}\text { Absorción masculina } \\
\text { Absorción femenina } \\
\text { Femenina de retorno }\end{array}$ & $\begin{array}{l}\text { Matrimonial de desarraigo } \\
\text { Expulsión } \\
\text { Estudiante }\end{array}$ \\
\hline
\end{tabular}

Fuente: Díaz Méndez (1997a).

${ }^{6}$ El análisis de las trayectorias de inserción femeninas ha sido desarrollado en profundidad por la autora considerando las particularidades de género en el proceso de inserción (Díaz Méndez, 1997b). 
Para su descripción vamos a dividir las estrategias familiares en dos bloques: por un lado, aquellas estrategias orientadas a apoyar el tránsito de la juventud a la vida activa dentro del grupo familiar, las denominadas trayectorias de continuación; por otro lado, aquellas orientadas a apoyar este tránsito fuera de la familia, las llamadas trayectorias de no continuación.

\subsection{TRAYECTORIAS DE CONTINUACión}

A este grupo pertenecen las trayectorias familiares orientadas a garantizar que uno de los hijos continúe en la explotación agraria. Estas trayectorias son las que más repercusiones tienen en todo el grupo, ya que el resto de las orientaciones parten de la consolidación de esta primera y de su aceptación por parte tanto del sucesor o sucesora como del resto de los hermanos y hermanas. La obligada presencia de un único hijo en las explotaciones agrarias estudiadas hace que la trayectoria de continuación se concentre en uno solo de ellos. La orientación variará dependiendo de que el hijo sucesor sea hombre o mujer, primando siempre la sucesión masculina.

El sucesor o sucesora puede rechazar esta opción y puede preferir una vía que le lleve hacia empleos no agrarios. La situación de dependencia familiar y laboral asociada al trabajo agrario familiar constituye la motivación más frecuentemente expresada por estos jóvenes para renunciar a su papel de sucesores. Los padres conocen esta posibilidad y retienen al joven ofreciéndole unas condiciones de vida que no le hagan sentirse diferente a los jóvenes no agrarios: la disponibilidad económica para el ocio, la compra de bienes y la mejora de las condiciones de la vivienda familiar son algunos de los aspectos que hacen esta alternativa laboral más atractiva para el sucesor frente a opciones de trabajo y de vida fuera de la familia.

Usando el género como criterio de distinción se pueden considerar las siguientes trayectorias:

\subsubsection{Trayectoria de absorción masculina}

En las familias que se apoyan en una explotación familiar moderna y de agricultura a tiempo parcial nos encontramos con una estrategia familiar dominante. Se guía a uno de los hijos hacia la sucesión. Se conforma como una trayectoria tradicional de continuidad a la que se ha denominado trayectoria de absorción masculina. Las familias generan pautas de acción que van absorbiendo progresivamente al joven sucesor hacia la explotación y hacia la familia, a la vez que se dan comportamientos de expulsión del resto de hermanos y hermanas. La sucesión es percibida por estos jóvenes sucesores como una 
alternativa posible y una opción laboral deseable, sobre todo en los últimos años, habida cuenta de la escasez de empleos no agrarios.

El momento de confirmación de la trayectoria laboral es la interrupción o el fracaso escolar en los estudios. La continuación del chico cuando sigue esta trayectoria se consolida con su implicación en la mejora de la explotación, implicación que le obliga a responsabilizarse, junto al padre, de la modernización de la misma. Las relaciones familiares cotidianas y el trabajo diario compartido van ofreciendo tanto al joven como al padre la posibilidad de sentirse colaboradores, asumiendo los jóvenes, de forma progresiva, responsabilidades laborales y familiares.

\subsubsection{Trayectoria de sucesión afectiva}

Ciertamente, las limitaciones económicas de algunas explotaciones familiares, combinadas con los deficientes recursos del entorno en el que se ubican, hacen pensar en la imposibilidad de lograr la reproducción de las condiciones de vida de algunas familias y sus explotaciones. Pero todas las familias cuentan con algunos recursos, por escasos que éstos sean, y los manejan para lograr sus objetivos. Así, aun en condiciones adversas, las familias elaboran estrategias orientadas a mantenerse a lo largo del tiempo, encuentran fórmulas para la sucesión y en muchos casos logran que un hijo les sustituya y prosiga con la explotación familiar. Esto es lo que sucede en la trayectoria de sucesión afectiva.

Para entender la reproducción familiar de estas explotaciones es preciso remitirse a aspectos basados en vínculos afectivos y las mutuas responsabilidades adquiridas por hijos y padres a lo largo del tiempo, que sirven aquí para orientar la sucesión y hacer que un hijo repita la historia familiar.

Los padres y las madres lo presentan como una opción tomada voluntariamente por sus hijos sucesores, pero con frecuencia se hace depender el éxito de los otros hermanos de que uno de ellos acepte la sucesión. Ante esta situación, el chico termina por aceptar una vía que no ha elegido y se resigna a ella ante las dificultades, cada vez mayores, de encontrar alternativas laborales fuera del pueblo. Aun así, las limitadas condiciones de vida a las que se ven sometidas estas familias de agricultura de subsistencia y el rechazo que, como veremos en las trayectorias siguientes, tienen las mujeres a unirse sentimentalmente a estos jóvenes, nos hace pensar que este tipo de sucesión es una trayectoria de continuación que finaliza con la soltería de este sucesor y la consiguiente ruptura del ciclo de reproducción familiar.

\subsubsection{Trayectoria puente}

Esta trayectoria de tránsito es el tipo de vía adoptada por algunos jóvenes que, siguiendo una trayectoria de sucesión afectiva, encuentran una fórmula 
que combina su obligada presencia en la explotación familiar con la posibilidad de llevar a cabo sus deseos de independencia. Aquí el joven sucesor encuentra la posibilidad de realizar alguna actividad laboral no agraria en el mismo pueblo o en los pueblos cercanos. El joven se mueve entre la familia y un empleo no agrario temporal, de ahí la denominación de trayectoria puente.

Este joven tenderá a rechazar los trabajos fijos. Su opción combina la familia y el trabajo externo. No romperá su vínculo familiar y seguirá manteniendo una implicación laboral con la explotación; sus padres, ya de edad avanzada, realizan el trabajo básico en su ausencia y dependen de él para los trabajos más duros. No romperá tampoco el vínculo con el trabajo externo, pues tanto los aspectos económicos de este segundo empleo como las relaciones externas al pueblo le resultan necesarios; de hecho, es la única posibilidad del joven de encontrar esposa.

\subsubsection{Trayectoria de absorción femenina}

Esta trayectoria está asociada, fundamentalmente, a las familias con explotaciones que se mantienen en un grado medio de competitividad que les permiten dar respuesta a las exigencias del mercado lácteo y mantenerse a pesar de la crisis del sector. Estas familias necesitan para mantenerse una persona joven dispuesta a trabajar y a sacar adelante una explotación que habitualmente se endeuda para hacerse competitiva.

Las mujeres jóvenes, hijas de estos ganaderos, no son consideradas, ni se consideran a sí mismas, como candidatas a la sucesión de la explotación familiar. La familia absorbe a una mujer para la sucesión sólo en aquellas familias en las que no hay hijos varones.

El matrimonio es la opción femenina por excelencia y se trata de una trayectoria que se consolida entorpeciendo a la mujer la alternativa de los estudios.

Para comprender la importancia de la mujer como esposa de ganadero es preciso diferenciar los dos tipos de roles que se han encontrado en las familias estudiadas: las esposas de ganaderos modernos y las esposas de ganaderos a tiempo parcial.

En las áreas con explotaciones más empresarializadas las estrategias de la familia se concentran en hacer de la chica una joven casadera; sin embargo, su papel una vez cumplida la misión matrimonial pasa a un segundo plano. Cada vez se encontrará más alejada del ganado y de las tierras y más vinculada a las tareas del hogar.

En las familias con explotaciones a tiempo parcial el rol doméstico no es el rol femenino más característico. En estas familias los padres trabajan en empleos no agrarios que compatibilizan con las tareas en las explotaciones familiares ayudados por sus esposas y otros familiares. Esta situación hace imprescindible la presencia de una mujer que sustituya al marido en el trabajo agrario y ganadero durante su ausencia. 


\subsubsection{Trayectoria femenina de retorno}

A la hora de realizar un análisis global de las trayectorias femeninas hemos encontrado a un grupo de familias con mujeres jóvenes al cargo del hogar, mujeres solteras que han regresado de la ciudad para hacerse cargo del cuidado de la familia. Las particulares características de estas jóvenes merecen un estudio detallado por las implicaciones que este retorno tiene para ellas y por ser el único modelo de trayectoria femenina en el que se observa con claridad algo frecuente en los modelos masculinos: la renuncia voluntaria a un objetivo individual en beneficio de la familia como grupo. Habitualmente es un suceso familiar dramático lo que las hace volver a la explotación y a la familia.

Las estrategias de orientación empleadas por las familias para guiar la inserción de estas mujeres coinciden con las de las mujeres con trayectorias de no continuación: apoyadas por la familia para abandonar, y prácticamente consolidada su vía de independencia urbana a través de los estudios o a través de algún empleo, regresan al hogar de origen para atender a sus familiares. Al margen de las circunstancias particulares, hay dos motivos por los que se reclama la vuelta de estas jóvenes: por ser mujeres y por estar solteras. Esta situación de dependencia de las mujeres solteras parece confirmar la percepción tradicional que se tiene de la independencia femenina en el mundo rural, una independencia que a la mujer se le otorga sólo a través del matrimonio.

Ninguna de las mujeres que hemos encontrado en esta situación considera que su futuro esté en el pueblo ni en la familia de origen, confían en que las cosas se resuelvan para reanudar las actividades abandonadas, lo perciben como una situación transitoria, siempre pendientes de la resolución de los problemas que las han traído de vuelta, esperan tiempos mejores.

\subsection{TRAYECTORIAS DE NO CONTINUACIÓN}

La familia, a la vez que elabora estrategias orientadas a absorber a un joven o una joven en la explotación familiar, genera otras orientaciones de expulsión en las que ofrece a los no sucesores distintos recursos para favorecer su tránsito a la vida activa y su instalación sociolaboral fuera de la familia agraria. Pasamos a continuación a describir cuatro tipos de trayectorias, dos masculinas y dos femeninas, que son resultado de una orientación familiar complementaria de la anterior.

Matrimonio y estudios van a ser los recursos utilizados para ello. Se encuentran también aquí variaciones en función del género. Estas trayectorias de no continuación son las que están más condicionadas por los recursos extrafamiliares, ya que las condiciones socioeconómicas del entorno en el que se ubican las explotaciones familiares van a determinar las posibilidades de empleo y formación de la juventud que no va a continuar con la explotación agraria familiar. 
Los estudios suelen ser aquí la alternativa más valorada y el recurso más conflictivo en el reparto. El diferente valor de las alternativas en áreas en las que las explotaciones son de baja rentabilidad y en las que los estudios ofrecen un soporte laboral de interés constituye la base del conflicto. El conflicto, sin embargo, aunque presente en algunas familias, parece estar resuelto al realizar los padres y las madres una distribución sexista de los recursos formativos y, así, la orientación hacia los estudios es una estrategia familiar que afecta preferentemente a las chicas.

\subsubsection{Trayectoria de expulsión masculina}

Asociada fundamentalmente a familias con una explotación a tiempo parcial o modernizada, esta vía se inicia con el intento de los padres y las madres de apoyar la continuación en los estudios de los jóvenes no destinados a la sucesión. Tras finalizar la formación básica les animan a proseguir con los estudios, en un intento de asegurar con estos recursos el futuro de los hombres no sucesores. Pero esta opción no suele tener éxito. El fracaso escolar disuade a estos chicos y a sus padres y madres de continuar por esta vía de inserción y la situación más frecuente es el inicio de estudios de tipo medio que son abandonados con un fracaso escolar ya anunciado en las etapas de escolarización obligatoria anteriores.

Curiosamente, esto se da incluso en las áreas rurales en las que las posibilidades formativas son amplias. A menudo los chicos inician las enseñanzas medias y las abandonan. Este fracaso escolar es particularmente dramático ya que así anulan la única alternativa que la familia les puede ofrecer para apoyar su inserción sociolaboral. La familia pondrá en movimiento sus relaciones parentales y de amistad para encontrar empleos para estos jóvenes. Estos recursos relacionales, sin embargo, no suelen ser suficientes para insertar a los jóvenes en empleos estables; la inestabilidad laboral del entorno y condicionados por una escasa o nula cualificación, son candidatos a empleos irregulares e inestables y permanecen en la familia, protegidos por ella, a la espera de su instalación fuera del hogar y en un trabajo no agrario. Mientras permanezcan en la familia se espera de ellos que colaboren en las tareas de la explotación, que permanezcan solteros y que no rechacen ninguna oferta laboral que les ofrezca una mínima posibilidad de independizarse del grupo.

\subsubsection{Trayectoria de nostalgia}

Hemos denominado así a una vía masculina caracterizada por la dependencia afectiva de los chicos hacia la familia de origen desde su posición de trabajadores en empleos no agrarios. Los jóvenes que han seguido esta trayectoria proceden principalmente de familias de agricultura y minería y son en la 
actualidad mineros en áreas alejadas de sus pueblos de origen. Para estos chicos, trabajo significa fundamentalmente empleo asalariado.

En estas zonas ni los padres ni las madres han deseado la continuación en la actividad agrícola familiar. La familia campesina ha ido abandonando una ganadería de subsistencia a medida que se ha ido consolidando el trabajo en la mina de los hombres de la casa.

La particularidad de esta situación es que los hijos varones mantienen un fuerte vínculo familiar a través, precisamente, de la actividad ganadera de la familia. La transmisión de unos saberes inútiles en un área sin posibilidades agrarias ha servido, sin embargo, para establecer vínculos sentimentales entre los hijos y los padres y ha generado unas relaciones de dependencia afectiva que une a los jóvenes a unas ganaderías de subsistencia que sus padres se encargan de mantener.

La particularidad de este vínculo es que afecta sólo a los varones. Esto nos hace pensar que se trata de un tipo de transmisión semejante a la que se da en las familias que buscan sucesor. Se ha preparado a los chicos para la sucesión, pero en este caso es una sucesión imposible ya que las condiciones socioeconómicas del entorno la han hecho imposible. Se mantiene, sin embargo, la pauta cultural de la sucesión, el apego a la familia y a la actividad agraria que une al grupo.

\subsubsection{Trayectoria femenina de estudiante}

Esta trayectoria está asociada a todos los tipos de agricultura analizados y tiene una relación directa con las trayectorias laborales de los hermanos sucesores. Las madres optan por empujar a sus hijas hacia otras agencias socializadoras que les garanticen el aprendizaje de otros modelos femeninos con el objetivo de alejarlas del destino al que por su origen parecen orientadas. Los estudios son determinantes para que sus hijas logren la independencia, al tiempo que las transformarán en mujeres urbanas con estilos de vida distintos a los rurales.

Las opciones que las madres contemplan para los varones están siempre dentro del marco de la tradición campesina, sólo a las chicas se las quiere alejar de su destino tradicional. Toda la familia, incluidos los jóvenes varones, se concentra en la estrategia de apoyo a las hijas para hacer de ellas unas señoritas, garantizando su marcha del pueblo. Esta discriminación, que podría ser considerada por los jóvenes varones como un agravio comparativo, no es percibida así, ya que ellos se ven afectados por otro tipo de orientación que les hace ver el tratamiento dado a sus hermanas como natural.

El apoyo permanente de sus madres y la vivencia de un mundo urbano en el que cierta independencia y autonomía personal es posible son los dos elementos motivadores para que las chicas continúen los estudios, a pesar de las dificultades que éstos representan y de sus tropiezos escolares. Muchas de ellas permanecen en el sistema educativo aun con escaso éxito, otras optan por la 
salida a través del trabajo en la ciudad. A éstas, que también logran con ello una independencia de la familia de origen, les esperan empleos sin cualificación y rechazan cualquier alternativa que las haga retroceder en su camino de huida hacia la independencia. La mujer campesina tradicional atada a la tierra y al marido crea unas condiciones de vida familiar para evitar que sus hijas reproduzcan su historia.

\subsubsection{Trayectoria matrimonial de desarraigo}

Como queda patente en el modelo anterior, la intención de muchas madres es alejar a las jóvenes de un destino agrario. Algunas familias logran este objetivo enviando a las chicas a estudiar; pero esta vía no siempre es posible. Las limitaciones económicas de la familia, las limitaciones formativas del entorno extrafamiliar o el fracaso escolar de las chicas puede orientar su inserción hacia vías que, igualmente eficaces, tienen también por objetivo alejarlas del trabajo agrario y enviarlas a la ciudad. La alternativa más habitual a los estudios es el matrimonio con un joven dedicado a actividades no relacionadas con la agricultura y la ganadería.

En estas familias las chicas ven una salida a su situación, a la vez que una salida del pueblo, en el matrimonio con un trabajador industrial y urbano. Ensayada ya la vía de los estudios por familias que cuentan con recursos económicos para ello y tras el fracaso escolar, las madres retienen a sus hijas en la cocina y las alejan del trabajo agrario y ganadero. En algunos casos asumen roles domésticos y adquieren responsabilidades de atención y cuidado familiar durante la espera. Algunas de ellas rechazan abiertamente el casarse con jóvenes ganaderos. Otras se afianzan en su posición de ama de casa y, aunque conocen el trabajo femenino en el campo, esperan que el matrimonio no las desvincule de la cocina.

La vía matrimonial femenina es, sin embargo, más propia de las hijas de las familias sin recursos económicos para afrontar unos estudios. El matrimonio es, para estas jóvenes, sin duda, la vía más eficaz para situarse socialmente.

\section{CONCLUSIONES}

Se ha hecho referencia a los distintos tipos de transición a la vida activa de la juventud rural que arrancan de la acción estratégica familiar. Esta acción se orienta a partir de los recursos económicos del grupo y del entorno, y la familia distribuye estos recursos entre sus hijos e hijas generando con ello una diversidad de trayectorias de tránsito a la vida adulta y activa de esta juventud.

El margen de maniobra de la familia no es ilimitado (sus recursos tampoco lo son), pero no está absolutamente determinado por las limitaciones del entorno intrafamiliar o extrafamiliar. Con grandes o escasos recursos, las fami- 
lias campesinas tienen como objetivo último de la acción el mantenimiento, ampliación y reproducción del grupo familiar. En un entorno cambiante, con presiones que condicionan la marcha socioeconómica de las explotaciones familiares y con alternativas sociolaborales y culturales fuera de la familia, las familias campesinas actuales manejan sus recursos para intentar combinar reproducción y cambio. Las estrategias familiares, guiadas por pautas de comportamiento colectivo, generan efectos contrarios a los previstos y desestructuran el grupo familiar impidiendo su reproducción.

La particularidad de este proceso en la familia campesina moderna es que no siempre el objetivo de supervivencia y continuación del grupo será posible, bien porque los recursos no sean suficientes para lograrlo, bien porque existan diferencias en el seno del grupo familiar que enfrenten los objetivos de la familia con los de sus miembros individuales. En este proceso los jóvenes tienen al menos dos opciones: proseguir con la familia o abandonarla, como se ha mencionado; continuar o no continuar en el grupo familiar. Como hemos visto, la distribución de los recursos en el proceso no es igualitaria, las estrategias de acción orientadas a la continuación son preferentemente masculinas; en cambio, las estrategias de no continuación afectan en mayor medida a las mujeres. Pero la variedad de estrategias contiene pautas de selección, la familia realiza una primera selección guiada por el objetivo de reproducción del grupo y se organiza buscando que uno de los jóvenes se mantenga unido a ella. En una segunda selección los recursos se distribuyen en función del género, dando prioridad a las mujeres en la asignación de recursos no agrarios. Se ha podido comprobar cómo los procesos de selección se consolidan a través de comportamientos intrafamiliares que favorecen la eficacia de las estrategias de acción y garantizan la participación de los individuos en el objetivo común.

Es sabido que la familia campesina tradicional busca ante todo su reproducción, y busca mejorar, si es posible, sus condiciones de vida y trabajo; sin embargo, la lógica de su acción no era tan conocida. Con esta investigación se ha visto cómo el grupo familiar maneja sus recursos y se orienta colectivamente hacia el apoyo de las trayectorias de transición a la vida activa de los hijos y de las hijas. Quizás lo más llamativo de este proceso sea que en algunos casos las familias parecen lograr un sucesor que garantice su reproducción, pero, en ocasiones, las propias estrategias de absorción de los sucesores imposibilitan la continuidad futura del grupo. A pesar de todo ello, la aceptación de la orientación familiar y de su particular forma de entender el tránsito a la vida adulta de la juventud es un hecho constatado en las familias estudiadas. Por encima del rechazo a una situación no deseada, bien de continuador, bien de no continuador, los jóvenes y las jóvenes parecen asumir su papel en el grupo. El sentido colectivo de la orientación, en general, se mantiene y a través de las relaciones intrafamiliares se logra la aceptación de la orientación por parte de los miembros de la familia sin provocar desavenencias ni rupturas dramáticas.

Estas pautas de transición a la vida adulta de la juventud seguidas por las familias aquí analizadas representan diferentes modos de afrontar un problema 
decisivo para la continuidad del mundo rural: la incorporación de la juventud a la vida activa en un contexto de bloqueo del mercado laboral, particularmente el juvenil, y de crisis del sector agrario, y confirma la importancia social y cultural de las familias agrarias y de su peculiar organización interna. Se ha visto la flexibilidad laboral que permite este tipo de organización familiar y su capacidad de adaptación tanto a las variaciones del entorno como a las de la propia familia. Todo ello constituye una protección para la pequeña agricultura y para quienes dependen de ella, sometida a las no siempre previsibles variaciones del mercado. Del mismo modo, esta particular organización social que protege a los individuos, constriñe su comportamiento y bloquea con ello las iniciativas juveniles. El objetivo de reproducción que mantiene unido al grupo no beneficia por igual a todos sus miembros y algunos jóvenes se encuentran particularmente desfavorecidos en el reparto familiar de recursos.

Para terminar cabe insistir en la particularidad ya no sólo del tránsito de la juventud rural a la vida activa, sino también en su diversidad. La juventud rural no se enfrenta a la vida activa de forma homogénea; su inserción se caracteriza, precisamente, por la diversidad. Esta diversidad se pone de manifiesto en las múltiples trayectorias encontradas en el trabajo aquí presentado.

\section{BIBLIOGRAFÍA}

Bretón, V.; García Pascual, F., y Mateu González, J. (coords.) (1997): La agricultura familiar en España. Estrategias adaptativas y politicas agropecuarias, Lleida, Universitat de Lleida.

CARABAÑa, J. (1993): «Educación y estrategias familiares de reproducción», en Estrategias familiares, de Garrido Medina y Gil Calvo (eds.), Madrid, Alianza, pp. 37-47.

Casal, J.; Masjuan, J. M., y Planas, J. (1991): La inserción profesional y social de los jóvenes: itinerarios de transición entre los 14 y los 25 años, Barcelona, Instituto de Ciencias de la Educación, Universidad Autónoma de Barcelona, CIDE, Ministerio de Educación y Ciencia.

CONSEJERÍA DE LA JUVENTUD (1989a): Estudio socioeconómico sobre el desarrollo comarcal y el empleo juvenil en la comarca noroccidental, Principado de Asturias, Consejería de la Juventud.

- (1989b): Estudio socioeconómico sobre el desarrollo comarcal y fomento del empleo juvenil en la comarca oriental, Principado de Asturias, Consejería de la Juventud.

- (1991): Estudio socioeconómico sobre el desarrollo comarcal y el empleo juvenil en la comarca suroccidental del Principado de Asturias: Allande, Cangas de Narcea, Degaña, Ibias y Tineo, Principado de Asturias, Consejería de la Juventud.

CONTRERAS, J. (1997): «Estrategias familiares de producción y reproducción», en La agricultura familiar en España. Estrategias adaptativas y políticas agropecuarias, de Bretón, García Pascual y Mateu González (coords.), Lleida, Universidad de Lleida.

Chayanov, A. V. (1974): La organización de la unidad económica campesina, Buenos Aires, Nueva Visión.

Díaz Méndez, C. (1997a): Estrategias familiares y juventud rural, Madrid, Ministerio de Agricultura, Pesca y Alimentación.

- (1997b): «Modelos de inserción sociolaboral de las jóvenes rurales», en PAPERS. Revista de Sociología, núm. 54 (en prensa).

GABINETE DE EsTUdios JUVENILES (1960): Encuesta sobre presupuestos mentales de la juventud española, Madrid, Delegación Nacional de Juventud.

GALESKI, B. (1977): Sociología del campesinado, Barcelona, Península. 
Garrido Medina, L., y Gil Calvo, E. (1993): «El concepto de estrategias familiares», en Garrido Medina y Gil Calvo (eds.), Estrategias familiares, Madrid, Alianza, pp. 13-34.

GONZÁleZ, J. J. (1990): «La incorporación de los jóvenes a la agricultura», en Revista de Estudios Agrosociales, núm. 154, pp. 39-96.

- (1993): «Efectos perversos de las estrategias familiares en la agricultura», en Garrido Medina y Gil Calvo (eds.), Estrategias familiares, Madrid, Alianza Universidad, pp. 132-144.

GonZÁlez, J. J.; De LuCAS, A., y OrTí, A. (1985): Sociedad rural y juventud campesina. Estudio sociológico de la juventud 1984, Madrid, Ministerio de Agricultura, Pesca y Alimentación.

GonzÁlez, J. J., y Gómez Benito, C. (1997): Agricultura y sociedad en la España contemporánea, Madrid, Ministerio de Agricultura, Pesca y Alimentación, Centro de Investigaciones Sociológicas.

IbÁÑEZ, J. (1979): Más allá de la sociología. El grupo de discusión: técnica y crítica, Madrid, Siglo XXI.

LISÓN, C. (1971): Antropología cultural de Galicia, Madrid, Siglo XXI.

Ministerio De EdUCACIÓN y CienCIa (1991): Equipamientos y guia de la Administración del Estado en Asturias, 1990, Principado de Asturias, Delegación del Gobierno en Asturias.

Moyano Estrada, E., y FernÁndeZ DuránteZ, M. C. (1990): "Teoría y práctica de la instalación de jóvenes en la agricultura», en Revista de Estudios Agrarios, núm. 154, octubrediciciembre 1990, pp. 7-38.

Newby, H., y SeVIlla GuZMÁn, E. (1983): Introducción a la sociología rural, Madrid, Alianza.

ORTí, A. (1986): «La apertura y el enfoque cualitativo o estructural: la entrevista abierta y la discusión de grupo", en GarCÍA FerRando y Alvira, El análisis de la realidad social. Métodos y técnicas de investigación, Madrid, Alianza Universal Textos, pp. 153-186.

PAlerM, A. (1980): Antropología y marxismo, México, Nueva Imagen.

Pérez Rivero, J. L. (1991): Aproximación a la dinámica de las explotaciones ganaderas asturianas, Oviedo, Tesis doctoral (inédita), Facultad de Ciencias Económicas y Empresariales, Universidad de Oviedo.

Rodríguez GutiérRez, F. (1991): «La organización del espacio rural asturiano: entre la tradición y la modernidad", en Encuentros Internacionales de Juventud, Cabueñes (inédito).

Segalen, M. (1980): Mari et femme dans la société paysanne, París, Flammarion.

Sevilla GuZmán, E. (1984): Sobre agricultores y campesinos, Madrid, Ministerio de Agricultura, Pesca y Alimentación.

SHANIN, T. (1976): Naturaleza y lógica de la economía campesina, Barcelona, Anagrama.

SADEI (1994): Campaña de saneamiento ganadero 1992, Principado de Asturias, Consejería de Agricultura.

Toharia, J. J., y García Ferrando, M. (1982): V encuesta nacional a la juventud 1982, Madrid, Dirección General de Juventud y Promoción Sociocultural.

ZÁrRAGA, J. L. de (1985): «Informe juventud de España. La inserción de los jóvenes en la sociedad", en Estudios de Juventud, núm. 1, pp. 205-229.

ZÁrragA, J. L. de, y CONDE, F. (1985): Encuesta OMNIBUS juventud 1984-1985, Madrid, Instituto de la Juventud.

\section{ABSTRACT}

The research is based on two academic fields, peasants' studies and peasant family studies. The approach allows us to get into the family milieu and the understanding of its inner logic. The research shows how both parents guide strategically their sons and daughters towards their productive life, in a way which makes possible the continuity of the peasant family. The context of the research is the Asturian region and family agriculture. A qualitative methodology based on collective analysis is used in order to observe a process which is born and developed within the family milieu. The instruments used include discussion groups and non-structured interviews. 